\title{
Ancestral Divergence of Rickettsia bellii from the Spotted Fever and Typhus Groups of Rickettsia and Antiquity of the Genus Rickettsia
}

\author{
DIANE R. STOTHARD, JONATHAN B. CLARK, † AND PAUL A. FUERST* \\ Department of Molecular Genetics, The Ohio State University, Columbus, Ohio 43210
}

\begin{abstract}
The eubacterial genus Rickettsia belongs to the $\alpha$ subgroup of the phylum Proteobacteria. This genus is usually divided into three biotypes on the basis of vector host and antigenic cross-reactivity characteristics. However, the species Rickettsia bellii does not fit into this classification scheme; this organism has characteristics common to both the spotted fever group and the typhus group biotypes and also exhibits some unique features. Sequences of the 16S rRNA and 23S rRNA genes from Rickettsia rickettsii (spotted fever group), Rickettsia prowazekii (typhus group), and $R$. bellii were studied to determine the position of $R$. bellii in the rickettsial classification scheme. The 23S rRNA gene sequences described in this paper are the first 23S rRNA sequences reported for any member of the Rickettsiaceae. The $23 S$ rRNA gene contains substantially more phylogenetic information than is contained in the 16S rRNA sequences, and the $23 \mathrm{~S}$ rRNA gene sequence has diverged about 1.9 times faster in the three Rickettsia species which we studied. Taken together, the molecular data obtained from the two genes indicate that $\boldsymbol{R}$. bellii is not a member of either the spotted fever group or the typhus group; rather, this organism appears to be the product of a divergence which predates the separation of the genus into the spotted fever group and the typhus group. Consequently, different combinations of the ancestral characteristics retained by $R$. bellii have been retained in the more derived lineages of the genus. A comparison of the 16S rRNA and 23S rRNA gene sequences of Rickettsia strains with other proteobacterial sequences confirmed that the genus Rickettsia is a unique deeply branching member of the $\alpha$ subgroup of the Proteobacteria and that the Rickettsia species form a monophyletic cluster. While divergence of the contemporary members of the genus Rickettsia occurred recently, the unique evolutionary line represented by this genus appears to be very old.
\end{abstract}

Rickettsia strains are small, gram-negative bacteria that are intimately associated with eukaryotic cells and are members of a diverse family, the Rickettsiaceae, which also includes other intracellular organisms, including the genera Ehrlichia, Wolbachia, Anaplasma, and Cowdria $(4,36)$. Rickettsiae have natural arthropod hosts (either ticks, mites, or insects) and can be pathogenic for humans and other vertebrates. The obligately intracellular lifestyle and fastidious nature of these organisms have made them difficult to study. A number of genotypic and phenotypic characteristics indicate that the Rickettsia species are closely related, and this genus is now recognized as the sole member of a branch of the $\alpha$ subgroup Proteobacteria (8) on the basis of $16 \mathrm{~S}$ rRNA cataloging and partial 16S rRNA gene sequence data (34).

The genus Rickettsia is divided into three biotypes on the basis of immunological cross-reactivity and ecological characteristics (36). Rickettsia species are placed into the spotted fever group (SFG), the typhus group (TG), or the scrub typhus group on the basis of several phenotypic criteria. Most recognized species belonging to the SFG are found in ixodid ticks; the only exception is Rickettsia akari, which is mite transmitted. These rickettsiae have also been defined antigenically by the presence of a shared major group antigen (11). Several tickborne SFG species that occur in North America have been identified. Rickettsia rickettsii, the etiological agent of Rocky Mountain spotted fever, is the type species of the SFG and is

\footnotetext{
* Corresponding author. Mailing address: Department of Molecular Genetics, The Ohio State University, 484 W. 12th Avenue, Columbus, OH 43210-1292. Phone: (614) 292-6403. Fax: (614) 292-4466. Electronic mail address: pfuerst@magnus.acs.ohio-state.edu.

$\dagger$ Present address: Department of Ecology and Evolutionary Biology, University of Arizona, Tucson, AZ 85721.
}

the most extensively studied species. A number of less pathogenic or nonpathogenic organisms have also been identified (8). The TG, whose members occur predominantly in the Old World, includes two insect-borne species, Rickettsia prowazekii and Rickettsia typhi, which are similar in ecology, ultrastructure, serology, and human pathogenicity, and one enigmatic tick-borne species, Rickettsia canada. The scrub typhus group is composed of several antigenically defined mite-borne strains belonging to the species Rickettsia tsutsugamushi which is confined to Asia. This species has not been studied as intensively as the other groups, and its relationship to other members of the genus is problematic. In fact, $R$. tsutsugamushi is so distinct that some workers have suggested that it should be reclassified in another bacterial genus (32). While the three Rickettsia biotypes are very distinct from each other, a few organisms classified in the genus Rickettsia do not fall distinctly into any of the biotypes. One of these ambiguous forms is the focus of this report.

Rickettsia bellii is a tick-borne organism that occupies an ambiguous position in the current classification scheme. A putative nonpathogen, $R$. bellii exhibits limited antigenic crossreactivity with both SFG and TG rickettsiae $(1,22)$. Morphologically, it is similar to SFG strains and is capable of intranuclear growth (22), a characteristic also found in SFG species. However, the $\mathrm{G}+\mathrm{C}$ content of $R$. bellii is $30 \mathrm{~mol} \%$ (22), a value more similar to $\mathrm{TG} \mathrm{G}+\mathrm{C}$ contents than to SFG G+C contents. Although $R$. bellii is antigenically cross-reactive with both SFG and TG species, serotyping data (determined by microimmunofluorescence) and the results of an analysis of proteins by sodium dodecyl sulfate-polyacrylamide gel electrophoresis (SDS-PAGE) suggested that this organism is clearly distinct from both SFG and TG rickettsiae (22). One of the goals of this investigation was to compare these phenotypic character- 
istics with molecular data for both the $16 \mathrm{~S}$ and $23 \mathrm{~S}$ rRNA genes.

In this paper we describe the complete $16 \mathrm{~S}$ ribosomal DNA sequence of $R$. bellii. Nearly complete sequences of the $16 \mathrm{~S}$ rRNA genes of $R$. rickettsii and $R$. prowazekii have been described previously (34). In addition, the complete or nearly complete 16S rRNA gene sequences of several other Rickettsia species or Rickettsia-like organisms have been determined recently $(30 \mathrm{a}, 37)$. However, even when SFG species are compared with TG species, the numbers of sequence differences found in the 16S rRNA gene in the genus Rickettsia are quite small (34). Therefore, we also obtained data for the large-subunit rRNA gene to help clarify the ambiguous evolutionary relationships between $R$. bellii and the SFG and TG species. The 23S rRNA gene sequences of $R$. bellii, $R$. rickettsii, and $R$. prowazekii which we determined are the first largesubunit rRNA sequences obtained for members of the Rickettsiales. The molecular data presented in this paper bear on the placement of $R$. bellii. In addition, our analysis is one of the first attempts to correlate phylogenetic changes in the largeand small-subunit rRNA genes within a bacterial genus. Our molecular data indicate that the large-subunit rRNA gene contains substantially more phylogenetic information than the $16 \mathrm{~S}$ rRNA gene. Using the $16 \mathrm{~S}$ and $23 \mathrm{~S}$ rRNA gene sequences, we determined that $R$. bellii is the product of an early divergence which occurred prior to the schism between the SFG and TG. While some other workers have suggested that this species should occupy a peripheral position $(10,26,27)$, we describe the first quantitative classification of $R$. bellii in which genes with known evolutionary patterns were used. In addition, our data provide insight into more extensive proteobacterial comparisons; they indicate that the genus Rickettsia is a distinct, ancient lineage of the $\alpha$ subgroup of the Proteobacteria.

\section{MATERIALS AND METHODS}

Strains. Rickettsial strains were obtained from the collection of the Vector-Borne Disease Unit, Ohio Department of Health, which is now housed in the Department of Molecular Genetics of The Ohio State University. The strains used in this study were $R$. bellii G2D42 and 369-C (22), $R$. rickettsii Sawtooth, (5) and $R$. prowazekii Madrid 22-2 (7).

Nucleic acid preparation and genomic cloning of the $16 \mathrm{~S}$ rRNA gene. Rickettsiae were extracted from Vero host cells by host cell trypsinization, distilled water osmotic lysis, differential centrifugation of the lysate, and Sephacryl S-1000 chromatography (25). The 16S rRNA gene of $R$. bellii 369-C was cloned in a bacterial plasmid for analysis. Other sequences were obtained by using the PCR (28). $R$. bellii genomic DNA was digested with restriction endonuclease HindIII and was transferred to nitrocellulose by the method of Southern (30). A single $3.1-\mathrm{kb}$ band was identified by using a nick-translated $16 \mathrm{~S}$ rRNA gene clone from Rochalimaea quintana (pMD24; a gift from M. Dobson) as the probe. This band and adjacent bands were isolated from an agarose gel. The fragments were then ligated into plasmid vector Bluescribe (Stratagene, La Jolla, Calif.) which had been cut with HindIII and treated with calf intestine alkaline phosphatase. Following transformation of competent Escherichia coli cells, colonies were screened by using the 16S rRNA Rochalimaea quintana probe and colony hybridization (13).

Primer design. Four sets of nucleotide primers that were used for PCR amplification of the 16S and 23S rRNA genes were constructed and used in combinations, as shown in Fig. 1. The primers were designed by considering known conserved regions in the $16 \mathrm{~S}$ and $23 \mathrm{~S}$ rRNA genes of several members of
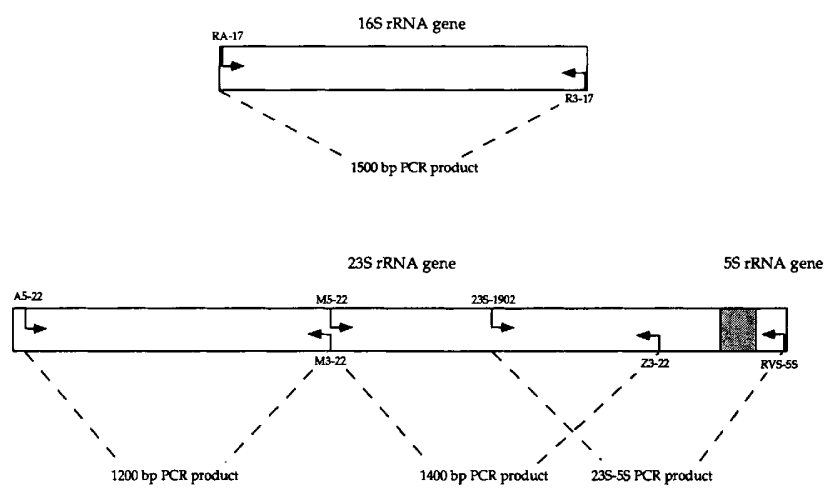

FIG. 1. Scheme for amplification of $16 \mathrm{~S}$ and $23 \mathrm{~S}$ rRNA genes, including location and identification of amplification primers. The shaded region in the schematic diagram of the $23 \mathrm{~S}$ rRNA gene represents the 23S-5S rRNA internal transcribed spacer.

the Proteobacteria. Primers RA-17 (5'-GGCTGCAGTCGAC GTTTGATCCTGGCTCAG-3') and R3-17 (5'-CCAGATCT GAGCTCAAGGAGGTGATCCAGCC-3') were designed to amplify the entire 16S rRNA gene in Rickettsia strains. Amplification of the 23S rRNA gene in Rickettsia strains as a single unit did not produce satisfactory yields. Therefore, the gene was amplified in three sections, as shown in Fig. 1, by using the following pairs of primers: A5-22 (5'-GGCTGCAGTCGACA GAGGCGATGAAGGACGTG-3') and M3-22 (5'-CCAGAT CTGAGCTCTCAGCATTCGCACTTCT-3'); M5-22 (5'-GG CTGCAGTCGACAGAAGTGCGAATGCTGA-3') and Z3-22 (5'-CCAGATCTGAGCTCTCCGGTCCTCTCGTACTAGG$\left.3^{\prime}\right)$; and 23S-1902 (5'-CGGCGGCCGTAACTATAACGG-3') and RVS-5S (5'-GGTCGACAGATCTAAGCCTGGCGG CGACTTAC- $3^{\prime}$ ). The last pair of primers was designed to amplify the $3^{\prime}$ end of the $23 \mathrm{~S}$ rRNA gene together with the 23S-5S rRNA transcribed spacer and the 5S rRNA gene of Rickettsia strains. SalI and PstI restriction sites were engineered into A5-22 and M5-22, BglII and SstI restriction sites were engineered into M3-22 and Z3-22, and SalI and BglII restriction sites were engineered into RVS-5S. These sites were added to the primers to help with asymmetrical cloning of amplification products into M13 vectors. An internal EcoRI restriction site was used in conjunction with the engineered site in RVS-5S to clone the 23S-5S ribosomal DNA product.

Several internal primers were designed for use in sequencing both the $16 \mathrm{~S}$ and $23 \mathrm{~S}$ rRNA genes. Again, these primers were designed by using conserved regions in the genes that had been identified on the basis of several previously described proteobacterial $16 \mathrm{~S}$ and $23 \mathrm{~S}$ rRNA gene sequences or on the basis of sequences obtained during the analysis of the rickettsial genes. The $16 \mathrm{~S}$ rRNA gene sequencing primers and their approximate locations were as follows: $16 \mathrm{~S}-400$ (located at position 371 in $E$. coli) (5'-CAATGGGCGAAAGCCTGATC C-3'); 16S-R400 (located at position 344 in E. coli) (5'-CCC ACTGCTGCCTCCCG-3'); 5003-18 (located at position 519 in $E$. coli) (5'-GTATTACCGCGGCTGCTG-3') and its complement, R5003-18; 5004-20 (located at position 907 in $E$. coli) (5'-CCGTCAATTCCTTTGAGTTT-3') and its complement, R5004-20; and 2-20 (located at position 1181 in $E$. coli) (5'-GACTTGACGTCATCCCCACC-3') and its complement, R2-20. The 23S rRNA gene sequencing primers and their approximate locations were as follows: A5-22 (located at position 519 in E. coli); M5-22 (located at position 1240 in $E$. coli) and its complement, M3-22; Z3-22 (located at position 
2640 in $E$. coli); $23 \mathrm{~S}-413$ (located at position 413 in E. coli) (5'-CCATCCTCCAAGGCTAAATAC-3'); RVS-450 (located at approximately position 450 in $E$. coli) (5'-CCTTTCCCT CACGGTACTTGTTC-3'); 23S-817 (located at position 817 in $E$. coli) (5'-CGAAAGCTATTTAGGTAGCGCC-3'); $23 \mathrm{~S}$ 1145 (located at approximately position 1145 in E. coli) (5'-GG GGCTCAAGTCATGTACCG-3') and its complement, RVS1145; 23S-1636 (located at position 1636 in $E$. coli) (5'-TACC AAGGCGCTTGAGAGAAC- $3^{\prime}$ ) and its complement, RVS1636; 23S-1902 (located at position 1902 in E. coli) and its complement, RVS-1902; 23S-2413 (located at position 2413 in $E$. coli) (5'-GGGCCATCGCTCAACGGATCCCCGG-3') and its complement, RVS-2413; and 23S-2700 (located at approximately position 2700 in $E$. coli) (5'-GCCAACGCAG GCTGGGTAGC-3') and its complement, RVS-2700.

Amplification. The sequences of the 16S rRNA genes of $R$. bellii G2D42 and $R$. rickettsii and the 23S rRNA genes of all three rickettsial species were determined by using PCR-amplified products. Each PCR was performed with 25 to $50 \mathrm{pmol}$ of each primer, 100 to $150 \mathrm{ng}$ of genomic DNA, and 2.5 U of Taq polymerase (Perkin-Elmer Cetus). Optimization of PCR for the 23S ribosomal DNA gene required increasing the $\mathrm{MgCl}_{2}$ concentration to $4 \mathrm{mM}$. PCR was performed for 30 to 35 cycles consisting of $95^{\circ} \mathrm{C}$ for $1 \mathrm{~min}, 45$ to $50^{\circ} \mathrm{C}$ for $2 \mathrm{~min}$, and $72^{\circ} \mathrm{C}$ for 3 min. The PCR products were pipetted from beneath the mineral oil layer, the pipette tip was wiped clean of oil with 95\% ethanol, and the PCR solution was transferred to clean tubes.

Cloning of PCR products. The 23S rRNA gene PCR products were cleaved at the engineered $S a l I$ and $B g l I I$ restriction sites and cloned into Sall-BamHI-cleaved M13 vectors. The 23S-5S rRNA PCR product was cleaved with SalI and EcoRI and cloned into SalI-EcoRI-cut M13 vectors. Digested PCR products and vectors were gel purified prior to ligation by using the glass bead method performed with a GeneClean II kit (Bio 101, Inc.). Ligations were performed in $10-\mu$ l reaction mixtures containing $1 \mathrm{U}$ of T4 ligase (Bethesda Research Laboratories, Gaithersburg, Md.) and having a ratio of insert to vector of approximately $2: 1$. Reactions were carried out at $14^{\circ} \mathrm{C}$ for 4 to $12 \mathrm{~h}$, and $3 \mu \mathrm{l}$ of the ligation reaction mixture was used to transform competent $E$. coli $\mathrm{DH} 5 \alpha \mathrm{F}^{\prime}$ cells (16). After clear plaques were selected and grown, single-stranded and doublestranded DNAs were isolated and purified.

DNA sequencing. Nucleotide sequencing reactions with cloned material were performed by the dideoxy chain termination method (29), using a Sequenase II kit (US Biochemical, Cleveland, Ohio). For some parts of the sequence, clones were difficult to obtain in one or both orientations. In these situations, direct dideoxy sequencing of PCR products was performed by using a cycle sequencing kit (Bethesda Research Laboratories) as described by the manufacturer. The $16 \mathrm{~S}$ rRNA genes of $R$. rickettsii and $R$. bellii G2D42 and the 23S rRNA gene of $R$. prowazekii were sequenced in this manner. To assess sequence accuracy, all amplified genes were sequenced from multiple PCR products and/or clones. We determined at least $80 \%$ of all sequences on both strands, and rRNA secondary structures were determined to evaluate potential base mismatches in stems which would suggest possible sequencing errors.

Nucleotide sequence alignment. Sequences were aligned manually by using the program ESEE (6), after we identified homologous positions on the basis of previously accepted rRNA secondary structures $(14,15)$. In analyses involving only Rickettsia species, all sites could be aligned unambiguously. In analyses in which other members of the Proteobacteria were involved, sites for which homology was ambiguous were excluded.

Additional taxa. The sequences of several members of the Proteobacteria whose 16S and 23S rRNA gene sequences were available were compared with the sequences of Rickettsia species. These organisms were $E$. coli, Pseudomonas cepacia, Rhodobacter capsulatus, and Rhodobacter sphaeroides. Bacillus subtilis, a gram-positive bacterium, was used as an outgroup in both the $16 \mathrm{~S}$ and $23 \mathrm{~S}$ rRNA analyses; the sequences used were sequences from the $r m B$ operon of $B$. subtilis.

Phylogenetic analysis. Once the sequences were aligned, the corrected levels of nucleotide divergence of the sequences were calculated by using the DNADIST program in PHYLIP version 3.1 (9) and the Kimura two-parameter correction. The branching pattern of the taxa was estimated from the distance matrix by the neighbor-joining method, using the NEIGHBOR program as implemented in PHYLIP. In addition, a cladistic reconstruction of the phylogenetic relationships among taxa was performed by using the parsimony program PAUP version 3.1.1 (31). In comparisons of the eight-species data set, only homologous sites for which alignment of all sequences was unambiguous were included in the analysis. This resulted in the use of 1,303 bases of the 16S rRNA gene and 2,701 bases of the 23S rRNA gene.

Nucleotide sequence accession numbers. The GenBank accession numbers for the nucleotide sequences determined in this study are as follows: $R$. bellii $16 \mathrm{~S}$ rRNA, U11014; $R$. bellii 23S rRNA, U11015; $R$. rickettsii 16S rRNA, U11021; $R$. rickettsii $23 \mathrm{~S}$ rRNA, U11022; and $R$. prowazekii $23 \mathrm{~S}$ rRNA, U11018. The GenBank accession numbers for the other nucleotide sequences used in this study are as follows: $E$. coli $16 \mathrm{~S}$ rRNA gene, J01695; E. coli $23 \mathrm{~S}$ rRNA gene, J01695; $P$. cepacia 16S rRNA gene, M22518; $P$. cepacia 23S rRNA gene, X16368; Rhodobacter capsulatus 16S rRNA gene, M60671; Rhodobacter capsulatus 23S rRNA gene, X06485; Rhodobacter sphaeroides 16S rRNA gene, X53853; Rhodobacter sphaeroides 23S rRNA gene, X53853; and B. subtilis $r m B$ operon, K00637.

\section{RESULTS}

16S rRNA gene sequences. Rickettsia species appear to have only a single rRNA operon. Only one amplification product resulted from use of the RA-17-R3-17 flanking primer set with Rickettsia species. Restriction digests obtained by using HindIII and the genomic DNAs of 12 Rickettsia species, including $R$. bellii, $R$. prowazekii, and $R$. rickettsii, produced single bands, which was consistent with the presence of a single copy of the 16S rRNA gene in all taxa, when they were probed with the $16 \mathrm{~S}$ ribosomal DNA clone from Rochalimaea quintana(pMD24). No HindIII site was identified in the previously described sequences of $R$. rickettsii, $R$. prowazekii, and $R$. typhi (34). Single-band patterns consistent with the hypothesis that there are single copies of the 16S rRNA gene in $R$. bellii, $R$. prowazekii, and the SFG species Rickettsia montana were also obtained when genomic DNA restricted by either SphI or EcoRI was probed by using labeled $R$. bellii $16 \mathrm{~S}$ rRNA. Results obtained previously for $R$. prowazekii also indicated that this organism has a single copy of the 16S rRNA (21).

The sequence of the cloned $16 \mathrm{~S}$ rRNA gene of $R$. bellii is 1,501 nucleotides long. This sequence is the first complete sequence of the 16S rRNA gene for any species of the genus Rickettsia. We detected no size difference between the PCR products of the $R$. bellii and $R$. rickettsii 16S rRNA genes. The sequence of the $16 \mathrm{~S}$ rRNA gene determined for $R$. rickettsii agrees with the sequence described in a previous report (34), 
TABLE 1. Levels of similarity and evolutionary distances between $16 \mathrm{~S}$ rRNA gene sequences

\begin{tabular}{|c|c|c|c|c|c|c|c|c|}
\hline \multirow[b]{2}{*}{ Species } & \multicolumn{8}{|c|}{ Level of sequence identity or avg no. of substitutions per position ${ }^{a}$} \\
\hline & 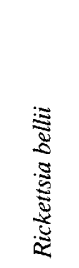 & 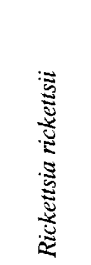 & 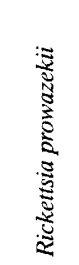 & 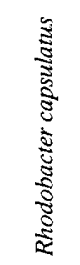 & 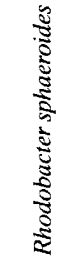 & 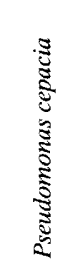 & 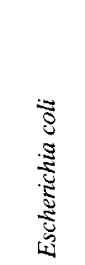 & 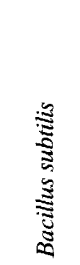 \\
\hline Rickettsia bellii & & 0.988 & 0.987 & 0.845 & 0.848 & 0.807 & 0.785 & 0.775 \\
\hline Rickettsia rickettsii & 0.012 & & 0.989 & 0.842 & 0.846 & 0.804 & 0.784 & 0.773 \\
\hline Rickettsia prowazekii & 0.013 & 0.010 & & 0.843 & 0.844 & 0.804 & 0.788 & 0.774 \\
\hline Rhodobacter capsulatus & 0.181 & 0.184 & 0.183 & & 0.957 & 0.793 & 0.779 & 0.777 \\
\hline Rhodobacter sphaeroides & 0.175 & 0.178 & 0.180 & 0.045 & & 0.802 & 0.792 & 0.778 \\
\hline Pseudomonas cepacia & 0.238 & 0.244 & 0.242 & 0.263 & 0.248 & & 0.832 & 0.779 \\
\hline Escherichia coli & 0.266 & 0.268 & 0.261 & 0.281 & 0.260 & 0.202 & & 0.770 \\
\hline Bacillus subtilis & 0.282 & 0.286 & 0.283 & 0.286 & 0.281 & 0.282 & 0.288 & \\
\hline
\end{tabular}

${ }^{a}$ The values on the upper right are the levels of fractional sequence identity within regions of unambiguous alignment, and the values on the lower left are the average numbers of substitutions per sequence position (evolutionary distances), adjusted as described by Kimura for multiple substitutions at individual positions.

except for clarification of 10 nucleotide positions at which ambiguities were found previously.

The corrected nucleotide difference values used in the phylogenetic comparison of the 16S rRNA gene sequences of the various taxa are summarized in Table 1 . The $16 \mathrm{~S}$ rRNA gene comparisons summarized in Table 1 represent 1,303 nucleotide sites which could be unambiguously aligned. The corrected nucleotide distances among the three Rickettsia species were very small compared with most other previously reported interspecific distances for prokaryotes; these distances were less than $1.4 \%$ for all three pairwise comparisons. If only the three Rickettsia species were compared, regions of ambiguous alignment were clarified, leaving 1,481 nucleotide sites for comparison, which included five sites that were variable in the three Rickettsia species and were not included in the eight-taxon analysis. At all five positions, $R$. prowazekii was different from $R$. bellii and $R$. rickettsii.

Analysis of the 16S rRNA gene data by either the neighborjoining method or the parsimony method produced the same tree (Fig. 2). This single short tree shows that $R$. bellii branched before the split of the SFG and TG species. In an exhaustive search for the shortest parsimony tree, using PAUP, we evaluated 10,395 trees. The most parsimonious $16 \mathrm{~S}$ rRNA tree required 796 mutational steps, while the next shortest tree required two additional changes. The latter tree connected $R$. rickettsii and $R$. bellii, with $R$. prowazekii forming a sister clade. The bootstrap values for the most parsimonious tree were $100 \%$ for all nodes except the node connecting $R$. rickettsii and

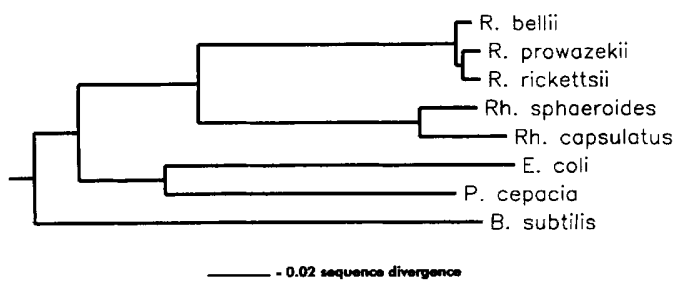

FIG. 2. Neighbor-joining tree based on 16S rRNA gene sequences. The distances from the terminal nodes along the horizontal branches are proportional to the corrected levels of nucleotide divergence. $\mathrm{Rh}$., Rhodobacter.
$R$. prowazekii, where the bootstrap value was $83 \%$. A tree joining $R$. rickettsii with $R$. bellii occurred only $17 \%$ of the time in the bootstrapped replicates.

23S rRNA gene sequences. The 23S rRNA gene of Rickettsia species includes approximately 2,760 base pairs. Because primer A5-22 is complementary to positions 42 to 60 in the $E$. coli $23 \mathrm{~S}$ rRNA sequence, the first 40 to 60 bases of the $23 \mathrm{~S}$ rRNA sequences of the three Rickettsia species are currently unknown. The terminus of the 23S rRNA genes of the Rickettsia species was located by analogy with the ends of the E. coli, P. cepacia, Rhodobacter capsulatus, and Rhodobacter sphaeroides genes.

A secondary structure for the 23S rRNAs of Rickettsia species was developed with the help of Michael Gray from Dalhousie University and will be available in the next version of the 23S rRNA database (15). This structure allowed us to verify certain parts of our sequence, and we compared this secondary structure with the secondary structures of the $23 \mathrm{~S}$ rRNA genes of other bacteria. Given the smaller database of complete or nearly complete large-subunit rRNA sequences compared with the 16S rRNA database, the existence of Proteobacteria group- or subgroup-specific structures is not yet obvious. However, the Rickettsia and Rhodobacter sequences differ from the sequences of other members of the Proteobacteria by the absence of approximately 120 bases in the region corresponding to $E$. coli stem structures 56 to 59 (approximately positions 1400 to 1600 in the $E$. coli sequence) and by a truncated stem produced by a deletion of approximately 15 bases corresponding to $E$. coli stem 63 (positions 1710 to 1750). There do not appear to be any major $23 \mathrm{~S}$ rRNA secondary structural features unique to the Rickettsia species which we examined.

The corrected nucleotide distances between 23S rRNA gene sequences are shown in Table 2. Again, the differences among the three Rickettsia species were small; these differences were only slightly larger on average than the differences found in the $16 \mathrm{~S}$ rRNA gene comparisons. The $23 \mathrm{~S}$ rRNA sequences of Rickettsia species exhibited about $2 \%$ divergence per nucleotide site. This value is also very small compared with data from other intrageneric comparisons. For instance, the two Rhodobacter species exhibited $8.6 \%$ divergence for the full $23 \mathrm{~S}$ rRNA comparison, in contrast to the $4.7 \%$ level of divergence 
TABLE 2. Levels of similarity and evolutionary distances between 23S rRNA gene sequences

\begin{tabular}{|c|c|c|c|c|c|c|c|c|}
\hline \multirow[b]{2}{*}{ Species } & \multicolumn{8}{|c|}{ Level of sequence identity or avg no. of substitutions per position ${ }^{a}$} \\
\hline & 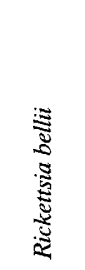 & 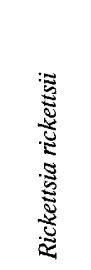 & 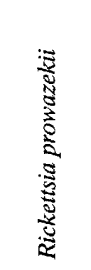 & 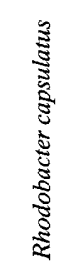 & 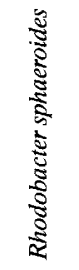 & 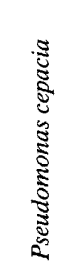 & 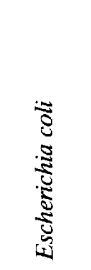 & 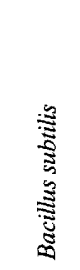 \\
\hline Rickettsia bellii & & 0.978 & 0.974 & 0.802 & 0.802 & 0.764 & 0.766 & 0.724 \\
\hline Rickettsia rickettsii & 0.022 & & 0.982 & 0.801 & 0.800 & 0.764 & 0.768 & 0.726 \\
\hline Rickettsia prowazekii & 0.027 & 0.018 & & 0.802 & 0.799 & 0.764 & 0.769 & 0.728 \\
\hline Rhodobacter capsulatus & 0.242 & 0.244 & 0.243 & & 0.930 & 0.788 & 0.778 & 0.737 \\
\hline Rhodobacter sphaeroides & 0.240 & 0.244 & 0.246 & 0.075 & & 0.786 & 0.780 & 0.728 \\
\hline Pseudomonas cepacia & 0.308 & 0.307 & 0.307 & 0.269 & 0.272 & & 0.831 & 0.753 \\
\hline Escherichia coli & 0.300 & 0.297 & 0.296 & 0.284 & 0.281 & 0.204 & & 0.735 \\
\hline Bacillus subtilis & 0.380 & 0.377 & 0.374 & 0.356 & 0.373 & 0.328 & 0.362 & \\
\hline
\end{tabular}

${ }^{a}$ The values on the upper right are the levels of fractional sequence identity within regions of unambiguous alignment, and the values on the lower left are the average numbers of substitutions per sequence position (evolutionary distances), adjusted as described by Kimura for multiple substitutions at individual positions.

observed when the full Rhodobacter $16 \mathrm{~S}$ rRNA gene sequences were compared. These data are some of the first comparative data concerning the relative rates of sequence divergence for the two complete rRNA genes in closely related bacterial species.

When we confined our analysis to the three Rickettsia species, we identified an additional 24 variable nucleotide positions that were removed from the eight-taxon comparison because they occurred in regions where alignment and homology were ambiguous. Four of these sites involved insertiondeletion events in which $R$. bellii appeared to have a deleted nucleotide. These four sites occurred between positions 530 and 540 in the Rickettsia gene. One other insertion-deletion event was located at position 645 in the gene, where $R$. bellii has a 2-nucleotide insertion. This region was included in the eight-taxon analysis.

Both the neighbor-joining method and the parsimony method produced the same topology for the Rickettsia species, clustering $R$. rickettsii with $R$. prowazekii and yielding a 23S rRNA phylogenetic tree (Fig. 3) almost identical to the 16S rRNA tree. The most parsimonious tree obtained by using an exhaustive search in PAUP required 1,991 steps, while the next most parsimonious tree was 5 steps longer. Again, the second most parsimonious tree grouped $R$. bellii and $R$. rickettsii, and $R$. prowazekii was a sister taxon. The bootstrap values for all nodes except the node connecting $R$. rickettsii and $R$. prowazekii were $100 \%$. The latter node was supported $88 \%$ of the time, while a tree joining $R$. rickettsii and $R$. bellii occurred in only

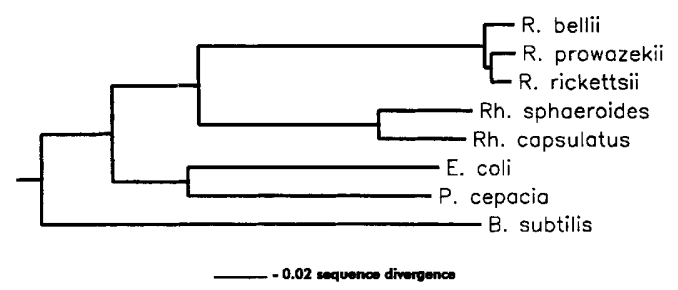

FIG. 3. Neighbor-joining tree based on 23S rRNA gene sequences. The distances from the terminal nodes along the horizontal branches are proportional to the corrected levels of nucleotide divergence. $R h$., Rhodobacter.
$12 \%$ of the bootstrapped replicates. As we found for the $16 \mathrm{~S}$ rRNA gene, the greatest corrected nucleotide distance found in the three pairwise comparisons occurred between $R$. bellii and $R$. prowazekii $(1.36 \%$ for the $16 \mathrm{~S}$ rRNA gene and $2.69 \%$ for the 23S rRNA gene).

Combined analysis. When the data for both genes were combined, the topology of the tree remained as shown in Fig. 2 and 3, with a joint bootstrap value of $89 \%$ supporting the joining of $R$. rickettsii and $R$. prowazekii. The second most parsimonious tree of the combined data set required seven additional mutational steps.

\section{DISCUSSION}

$R$. bellii shares morphological and immunological characteristics with both the SFG and the TG, which blurs its phylogenetic relationship to other rickettsiae. Cross-reactivity within the biotypes has been used as a major criterion to classify unknown Rickettsia strains as members of the scrub typhus group, the TG, or the SFG. Initial encounters with $R$. bellii led researchers to believe that it was an SFG species on the basis of its association with ixodid ticks and modest staining with a rabbit anti- $R$. rickettsii conjugate (23). $R$. bellii was also isolated in regions that were commonly geographic locales for $R$. rickettsii and Rocky Mountain spotted fever. However, the ability of $R$. bellii to cross-react antigenically with TG antisera and the TG-like $\mathrm{G}+\mathrm{C}$ content of its genome made phylogenetic inferences based on phenotypic data difficult.

When SDS-PAGE was used, the similarities in the electrophoretic patterns of TG and SFG strains were found to be greater than the similarities between members of either group and $R$. bellii (22). On the basis of phenotypic features, $R$. bellii was assigned to a unique biotype that was distinct from the SFG and the TG (22). However, it was suggested that this relationship could be clarified only with genetic data.

Our $16 \mathrm{~S}$ and 23S rRNA gene sequence data indicate that $R$. bellii is a member of neither the SFG nor the TG. Despite the small number of informative sites which differentiate the three Rickettsia species which we studied, the branching order of these species is well defined. $R$. bellii appears to have diverged shortly before the SFG-TG schism, making it distinct from contemporary SFG and TG species. This finding helps clarify several confusing characteristics of $R$. bellii. The inability of 
workers to easily classify $R$. bellii into either of these biotype groups can now be better understood. Common characteristics shared by the SFG or TG with $R$. bellii can be interpreted as ancestral characteristics which have been retained and do not indicate that $R$. bellii evolved as a novel biotype, as previously suggested (22).

Several other hypotheses concerning evolution within the genus Rickettsia are suggested by the finding that $R$. bellii represents a primitive group within the genus. The fact that $R$. bellii represents a phylogenetic line that predates the TG-SFG split suggests that the use of insects as primary hosts was a secondary event within the TG and that the genus Rickettsia is primarily associated with ticks. This is consistent with the more severe deleterious effects of TG organisms (with the possible exception of the recently described ELB agent [3]) on their insect arthropod hosts than of SFG organisms on their acarine hosts (35). Examination of the molecular placement of $R$. canada, a putative TG member which is associated with ticks, should provide further evidence about the movement of the TG into insects.

Finally, identification of $R$. bellii as a product of a primitive branch of the genus Rickettsia suggests further interpretations about the nature and origin of the genus. This genus contains both species that are pathogenic and species that are nonpathogenic in human populations, where pathogenicity is defined in terms of association with a disease phenotype in nonimmunocompromised individuals. $R$. bellii has never been isolated in conjunction with a disease phenotype and thus is considered nonpathogenic. Considering the basal position of this species in the genus, it is interesting to speculate that the pathogenic effects of some Rickettsia species must have arisen after the divergence of the primitive line leading to $R$. bellii from the line which gave rise to the TG and SFG.

The ancestral position of $R$. bellii also brings into question the suggestion that the genus originated in the Old World and subsequently migrated to the New World with rodent hosts (18). When care is taken to differentiate between different rickettsial forms, $R$. bellii is often found to be the most prevalent rickettsial species observed in ticks in North America (24). It has not yet been reported from Palearctic sources. Since $R$. bellii is apparently confined to North America (23), a Nearctic or Neotropical origin for the genus must be considered.

It is interesting to speculate about the possible time of divergence of the members of the genus Rickettsia. A very preliminary approximation can be obtained by using the molecular clock calibrations previously proposed for prokaryotic 16S-like small-subunit rRNA genes $(19,20)$, which suggested that $16 \mathrm{~S}$ rRNA nucleotide sequence divergence between two bacterial taxa occurs at a rate of about $1 \%$ per $50 \times 10^{6}$ years. On the basis of this estimate, the divergence between $R$. bellii and the SFG and TG would have occurred between $80 \times 10^{6}$ and $90 \times 10^{6}$ years ago. This is consistent with an association with the primitive ixodid tick hosts at the time of the mammalian radiation. Since ixodid ticks are confined to mammals, while argasid ticks are similarly confined to birds, which suggests that there was a major divergence in the acarine lineages about the middle Cretaceous (i.e., about $135 \times 10^{6}$ years ago) (17), a search for a close relative of the genus Rickettsia might focus on intracellular forms associated with argasid ticks. It is interesting that two strains of $R$. bellii have been isolated from argasid ticks (23), the only Rickettsia strains that have been obtained from argasid ticks.

The 16S and 23S rRNA phylogenetic trees in Fig. 2 and 3 show that the event leading to the separation of the genus Rickettsia from the genus Rhodobacter, the other $\alpha$ subgroup proteobacterial genus included in this analysis, occurred very soon (in terms of rRNA evolution) after the three major groups diverged from their common proteobacterial ancestor. In fact, the level of divergence between the genera Rickettsia and Rhodobacter is as great as the level of divergence between $E$. coli and $P$. cepacia, members of separate subgroups in the Proteobacteria (Tables 1 and 2). Thus, the genus Rickettsia seems to represent an extremely early diverging line within the $\alpha$ subgroup of the Proteobacteria (33). The apparent antiquity of the divergence of the genus Rickettsia from other members of the $\alpha$ subgroup of the Proteobacteria does not substantially change if one considers additional forms, such as the nearest available relatives of the genus Rickettsia, members of the genus Ehrlichia, for which only 16S rRNA gene sequences have been determined $(2,34)$. The average nucleotide distance for 16S rRNA sequences between three Rickettsia species and nine Ehrlichia species is $0.186 \pm 0.006$, a value slightly greater than the value obtained in this analysis for the distance between the genera Rickettsia and Rhodobacter $(0.180 \pm 0.003)$. The large divergence value which separates the genus Rickettsia and other members of the $\alpha$ subgroup of the Proteobacteria, such as the genus Ehrlichia, requires careful analysis, since it has been hypothesized that both the genus Ehrlichia and the genus Rickettsia are parts of a lineage which included the mitochondrial progenitor (12). A comparison of the genera Rickettsia and Ehrlichia with a broad range of other members of the $\alpha$ subgroup of the Proteobacteria and with mitochondrial sequences should include an analysis of $23 \mathrm{~S}$ rRNA gene data, which are not yet available for most of the relevant members of the $\alpha$ subgroup of the Proteobacteria, including Ehrlichia species. We are currently attempting to complete the sequence of the Ehrlichia risticii 23S rRNA gene to determine whether the hypothesis that there was an ancient rickettsial divergence is also supported by $23 \mathrm{~S}$ rRNA gene data.

At a finer level, while the genus Rickettsia diverged at an early stage from the other members of the $\alpha$ subgroup of the Proteobacteria, the rRNA data reveal that this genus is a monophyletic cluster, exhibiting very low levels of nucleotide divergence (Tables 1 and 2). If $R$. bellii continues to be the most divergent organism, no species will differ by more than about $1.2 \%$ in the $16 \mathrm{~S}$ rRNA gene and $2.6 \%$ in the $23 \mathrm{~S}$ rRNA gene, values which are quite small for most intrageneric comparisons in eubacteria. For instance, a comparison of the complete sequences of the genes of two Rhodobacter species revealed $8.6 \%$ divergence in the complete $23 \mathrm{~S}$ rRNA sequence and $4.7 \%$ divergence in the $16 \mathrm{~S}$ rRNA sequence.

The data from our analysis also provide information concerning the relative evolutionary rates of the two rRNA genes. Our comparisons are especially appropriate because of the limited divergence of the two genes, suggesting that little correction must be made in the estimated rates to account for multiple substitutions. The 23S rRNA gene in the genus Rickettsia has evolved 1.9 times faster than the 16S rRNA gene. For the genus Rhodobacter, the corresponding ratio of $23 \mathrm{~S}$ and $16 \mathrm{~S}$ rRNA evolutionary rates is 1.67 .

\section{ACKNOWLEDGMENTS}

We thank Mike Dobson for providing the Rochalimaea quintana $16 \mathrm{~S}$ rRNA probe, K. Poetter and C. Pretzman for growing rickettsial cultures, and workers at the Ohio Department of Health Vector-Borne Disease Unit (especially R. Berry and P. Parsons) for cooperating in our rickettsial studies and for allowing us to use their facilities for some of the preparation of bacterial cultures. We also thank Michael Gray for assistance with determining the secondary structures of the $23 \mathrm{~S}$ rRNA sequences, which helped us to clarify sequencing ambiguities.

This work was supported in part by a grant from the Office of Research of The Ohio State University. 


\section{REFERENCES}

1. Anacker, R. L., R. E. Mann, and C. Gonzales. 1987. Reactivity of monoclonal antibodies to Rickettsia rickettsii with spotted fever group and typhus group rickettsiae. J. Clin. Microbiol. 25:167-171.

2. Anderson, B. E., J. E. Dawson, D. C. Jones, and K. H. Wilson. 1991. Ehrlichia chaffeensis, a new species associated with human ehrlichiosis. J. Clin. Microbiol. 29:2838-2842.

3. Azad, A. F., J. B. Sacci, Jr., W. M. Nelson, G. A. Dasch, E. T. Schmidtmann, and M. Carl. 1992. Genetic characterization and transovarial transmission of a typhus-like rickettsia found in cat fleas. Proc. Natl. Acad. Sci. USA 89:43-46.

4. Brenner, D. J., S. P. O'Connor, H. H. Winkler, and A. G. Steigenerwalt. 1993. Proposals to unify the unify the genera Bartonella and Rochalimaea, with descriptions of Bartonella quintana comb. nov., Bartonella vinsonii comb. nov., Bartonella henselae comb. nov., and Bartonella elizabethae comb. nov., and to remove the family Bartonellaceae from the order Rickettsiales. Int. J. Syst. Bacteriol. 43:777-786.

5. Burgdorfer, W., T. R. Atkins, and L. E. Priester. 1975. Rocky Mountain spotted fever (tick-borne typhus), its agent, and its tick vectors in the United States. Am. J. Trop. Med. Hyg. 24:866-872.

6. Cabot, E. L., and A. T. Beckenbach. 1989. Simultaneous editing of multiple nucleic acid and protein sequences with ESEE. Comput. Appl. Biosci. 5:233-234.

7. Clavero, G., and F. Perez Gallardo. 1943. Estudio experimental de una cepa apatogenica y immunizante de Rickettsia prowazekii. Rev. Sanid. Hig. Publica 17:1-27.

8. Dasch, G. A., and E. Weiss. 1991. The genera Rickettsia, Rochalimaea, Ehrlichia, Cowdria and Neorickettsia, p. 2407-2469. In A. Balows, H. G. Trüper, M. Dworkin, W. Harder, and K. H. Schleifer (ed.), The prokaryotes, vol. 3, 2nd ed. Springer-Verlag, New York.

9. Felsenstein, J. 1989. PHYLIP—-phylogeny inference package (version 3.3). Cladistics 5:164-166.

10. Fuerst, P. A., and K, P. Poetter. 1991. DNA sequence differentiation in North American spotted fever group species of Rickettsia, p. 162-169. In J. Kazar and D. Raoult (ed.), Rickettsiae and rickettsial diseases. Slovak Academy Sciences, Bratislava, Slovakia.

11. Gilmore, R. D., Jr., and T. Hackstadt. 1991. DNA polymorphism in the conserved $190 \mathrm{kDa}$ antigen gene repeat region among spotted fever group rickettsiae. Biochim. Biophys. Acta 1097:7780.

12. Gray, M. W. 1993. Origin and evolution of organelle genomes. Curr. Opin. Genet. Dev. 3:884-890.

13. Grunstein, M., and D. S. Hogness. 1975. Colony hybridization: a method for the isolation of cloned DNAs that contain a specific gene. Proc. Natl. Acad. Sci. USA 72:3961-3965.

14. Gutell, R. R. 1993. Collection of small subunit (16S and 16S-like) ribosomal RNA structures. Nucleic Acids Res. 21:3051-3054.

15. Gutell, R. R., M. W. Gray, and M. N. Schnare. 1993. A compilation of large subunit (23S and 23S-like) ribosomal RNA structures. Nucleic Acids Res. 21:3055-3074.

16. Hanahan, D. 1983. Studies on transformation of Escherichia coli. J. Mol. Biol. 166:557-569.

17. Hoogstraal, H. 1978. Biology of ticks, p. 3-32. In J. H. K. Wilde (ed.), Tick-borne diseases and their vectors. Edinburgh University Press, Edinburgh.

18. Marchette, N. J. 1982 . The tickborne rickettsiae of the spotted fever group or tick typhus group, p. 75-112. In N. J. Marchette (ed.), Ecological relationships and evolution of the rickettsiae, vol. 1. CRC Press, Inc., Boca Raton, Fla.

19. Moran, N. A., M. A. Munson, P. Baumann, and H. Ishikawa. 1993. A molecular clock in endosymbiotic bacteria is calibrated using the insect hosts. Proc. R. Soc. Lond. Biol. Sci. 253:167-171.

20. Ochman, H., and A. C. Wilson. 1987. Evolution in bacteria: evidence for a universal substitution rate in cellular genomes. J. Mol. Evol. 26:74-86.

21. Pang, H., and H. H. Winkler. 1993. Copy number of the $16 \mathrm{~S}$ rRNA gene in Rickettsia prowazekii. J. Bacteriol. 175:3893-3896.

22. Philip, R. N., E. A. Casper, R. L. Anacker, J. Cory, S. F. Hayes, W. Burgdorfer, and C. E. Yunker. 1983. "Rickettsia bellii" sp. nov.: a tick borne Rickettsia, widely distributed in the United States, that is distinct from the spotted fever and typhus biogroups. Int. J. Syst. Bacteriol. 33:94-106.

23. Philip, R. N., E. A. Casper, W. Burgdorfer, R. K. Gerloff, L. E. Hughes, and E. J. Bell. 1978. Serologic typing of rickettsiae of spotted fever group by microimmunofluorescence. J. Immunol. 121:1961-1968.

24. Pretzman, C., N. Daugherty, K. Poetter, and D. Ralph. 1990. The distribution and dynamics of Rickettsia in the tick population of Ohio. Ann. N.Y. Acad. Sci. 590:227-236.

25. Ralph, D., C. Pretzman, N. Daugherty, and K. Poetter. 1990. Genetic relationships among the members of the family Rickettsiaceae as shown by DNA restriction fragment polymorphism analysis. Ann. N.Y. Acad. Sci. 590:541-552.

26. Regnery, R. L., C. L. Spruill, and B. D. Plikaytis. 1991. Genotypic identification of rickettsiae and estimation of intraspecies sequence divergence for portions of two rickettsial genes. J. Bacteriol. 173:1576-1589.

27. Roux, V., and D. Raoult. 1993. Genotypic identification and phylogenetic analysis of the spotted fever group rickettsiae by pulsed-field gel electrophoresis. J. Bacteriol. 175:4895-4904.

28. Saikai, R. K., S. Scharf, F. Faloona, K. Mullis, G. Horn, H. A. Erlich, and N. Arnheim. 1985. Enzymatic amplification of betaglobin genomic sequences and restriction site analysis for diagnosis of sickle cell anemia. Science 230:1350-1354.

29. Sanger, F., S. Nicklen, and A. R. Coulson. 1977. DNA sequencing with chain-terminating inhibitors. Proc. Natl. Acad. Sci. USA 74: 5463-5467.

30. Southern, E. M. 1975. Detection of specific sequences among DNA fragments separated by electrophoresis. J. Mol. Biol. 98: 503-517.

30a.Stothard, D., and P. Fuerst. Evolutionary analysis of the spotted fever and typhus groups of Rickettsia using 16S rRNA gene sequences. Syst. Appl. Microbiol., in press.

31. Swofford, D. 1993. PAUP: phylogenetic analysis using parsimony, ver. 3.1.1. Illinois Natural History Survey, Champaign.

32. Tamura, A., H. Urakami, and N. Oshashi. 1991. A comparative view of Rickettsia tsutsugamushi and other groups of rickettsiae. Eur. J. Epidemiol. 7:259-269.

33. Weisberg, W. G. 1989. Polyphyletic origin of bacterial parasites, p. 1-16. In J. W. Moulder (ed.), Intracellular parasitism. CRC Press, Boca Raton, Fla.

34. Weisburg, W. G., M. E. Dobson, J. E. Samuel, G. A. Dasch, L. P. Mallavia, O. Baca, L. Mandelco, J. E. Sechrest, E. Weiss, and C. R. Woese. 1989. Phylogenetic diversity of the rickettsiae. J. Bacteriol. 171:4202-4206.

35. Weiss, E. 1992. rickettsias, p. 585-610. In J. Lederberg (ed.), Encyclopedia of microbiology, vol. 3. Academic Press, San Diego, Calif.

36. Weiss, E., and J. Moulder. 1984. The rickettsias, p. 687-729. In N. R. Krieg and J. G. Holt (ed.), Bergey's manual of systematic bacteriology, vol. 1. Williams and Wilkins, Baltimore.

37. Werren, J. H., G. D. D. Hurst, W. Zhang, J. A. J. Breeuwer, R. Stouthamer, and M. E. N. Majerus. 1994. Rickettsial relative associated with male killing in the ladybird beetle (Adalia bipunctata). J. Bacteriol. 176:388-394. 